\title{
Face Recognition for Smart Environments
}

Alex Pentland and Tanzeem Choudhury

\author{
Reprint from \\ IEEE Computer
}

February 2000

(C) 2000 IEEE. Personal use of this material is permitted. However, permission to reprint/republish this material for advertising or promotional purposes or for creating new collective works for resale or redistribution to servers or lists, or to reuse any copyrighted component of this work in other works must be obtained from the IEEE.

This material is presented to ensure timely dissemination of scholarly and technical work. Copyright and all rights therein are retained by authors or by other copyright holders. All persons copying this information are expected to adhere to the terms and constraints invoked by each author's copyright. In most cases, these works may not be reposted without the explicit permission of the copyright holder. 


\section{Face Recognition for Smart Envir onments

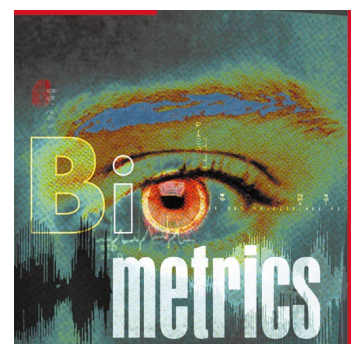

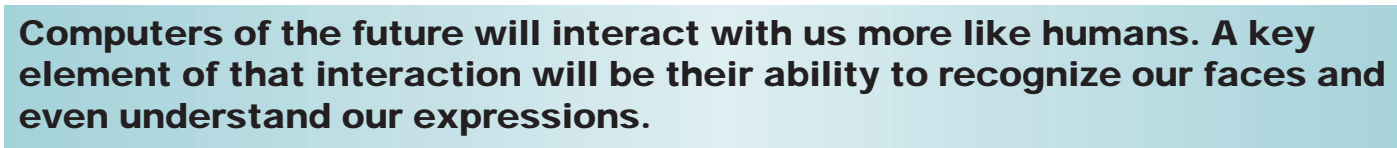

Alex (Sandy) Pentland

Tanzeem Choudhury

M IT M edia

Laboratory

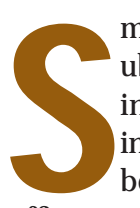
mart environments, wearable computers, and ubiquitous computing in general are the coming "fourth generation" of computing and information technology. ${ }^{1-3}$ These devices will be everywhere-clothes, home, car, and office - and their economic impact and cultural significance will dwarf those of the first threegenerations. At the very least, they represent some of the most exciting and economically important research areas in information technology and computer science.

But before this new generation of computers can be widely deployed, those working on interfaces must invent new methods of interaction without a keyboard or mouse. To win wideconsumer acceptance, these interactions must be friendly and personalized, which implies that next-generation interfaces will beaware of the peoplein their immediate environment and, at a minimum, know who they are.

\section{EANS OF IDENTIFICATION}

Given the requirement for determining people'sidentities, the obvious question is, what technology is best? A wide variety of identification technologies are available, and many have been in widespread commercial use for years. The most common personal verification and identification methods today are password/PIN (personal identification number) systems and token systems (using tokens such as your driver's license). Because such systems are vulnerable to forgery, theft, and lapses in users' memories, biometric identification systems, which use pattern recognition techniques to identify people by their physiological characteristics, are attracting considerable interest. Fingerprints are a classic example of a biometric; newer technologies include retina and iris recognition.

W hile they are appropriate for bank transactions and entry into secure areas, such biometric technolo- gies have the disadvantage of being intrusive, both physically and socially. They require users to position their bodies relative to the sensor and then pause for a second to "declare" themselves. This pause-anddeclare interaction is unlikely to change because of the fine-grained spatial sensing required. There is an "oracle-like" aspect to the interaction as w ell. Since people do not recognize each other by such things as retina scans, these types of identification feel intrusive.

The pause-and-declare interaction is useful in highsecurity applications (the interruption makes people security conscious), but it is exactly the opposite of what is required for a store that recognizes its best customers, an information kiosk that remembers you, or a house that knows the people who live there. Face and voice recognition have a natural place in these next-generation smart environments. They are unobtrusive (they recognize at a distance without a pause-and-declare interaction), they are usually passive (needing no special electromagnetic illumination), they do not restrict user movement, and they arenow both low power and inexpensive. Perhaps most important, though, is the fact that humans identify other people by their faces and voices and are likely to be comfortable with systems that use similar means of recognition.

\section{ACHIEVING FACE RECOGNITION}

Twenty years ago, the problem of face recognition was considered among the hardest in artificial intelligenceand computer vision. Surprisingly, however, over the past decade, a series of successes have made general personal identification appear not only technically feasible but also economically practical.

The apparent tractability of the face recognition problem, combined with the dream of smart environments, has produced a huge surge of interest from funding agencies and from researchers themselves. It 
has also spawned several thriving commercial enterprises. There are now several companies that sell commercial face recognition software that is capable of high-accuracy recognition with databases of more than 1,000 people.

These early successes came from the combination of well-established pattern recognition techniques with a fairly sophisticated understanding of the image generation process. In addition, researchers realized that they could capitalize on regularities that are peculiar to people. For instance, human skin colors lie on a one-dimensional manifold in color space, with color variation primarily due to melanin concentration. H uman facial geometry is limited and essentially twodimensional when peoplearelooking toward the camera. Today, researchers are working on relaxing some constraints of existing face recognition algorithms to achieve robustness under changes due to lighting, aging, rotation in depth, and expression. They are also studying how to deal with variations in appearance due to such things as facial hair, glasses, and makeup-problems that already have partial solutions.

Typical representational fr amew or $k$

The dominant representational approach that has evolved is descriptive rather than generative. This approach uses training images to characterizetherange of two-dimensional appearances of objects the system must recognize. Although researchers initially used very simple modeling methods, they now principally characterize appearance by estimating the probability density function (PDF) of theimage data for the target class.

For instance, given several examples of a target class $\Omega$-for example, faces-in a low-dimensional representation of the image data, it is straightforward to model the PDF $\mathrm{P}(\mathrm{x} \mid \Omega)$ of its image-level features $\mathrm{x}$ as a simple parametric function-a mixture of G aussian distribution functions-thus obtaining a low-dimensional, computationally efficient appearance model for the target class. In other words, we can use example face images to obtain a simple mathematical model of facial appearance in image data.

O nce wehavelearned thePDF of thetarget class, we can use Bayes' rule to perform maximum a posteriori (M AP) detection and recognition. The result istypically a very simple, neural-net-like representation of the target class's appearance, which a system can use to detect occurrences of the class, to compactly describe its appearance, and to efficiently compare different examples from the same class. Indeed, this representational framework is so efficient that some current face recognition methods can process video data at 30 frames per second. Several systems can comparean incoming face to a database of thousands of people in under one second- and all on a standard PC!
Dealing with dimensionality

To obtain an appearance-based representation, the image must first be transformed into a low-dimensional coordinate system that preserves the general perceptual quality of the target object's image. This transformation is necessary to address the problem of dimensionality: The raw image data has so many degrees of freedom that it would require millions of examples to directly learn the range of appearances. Typical methods for reducing dimensionality include

- Karhunen-Loève transform (also called principal components analysis),

- Ritz approximation (also called example-based representation),

- Sparse-filter representations (for example, Gabor jets and wavelet transforms),

- Feature histograms, and

- Independent-component analysis.

These methods all allow efficient characterization of a low-dimensional subspace within the large space of raw image measurements. O nce you obtain a lowdimensional representation of the target class-face, eye, or hand-you can use standard statistical parameter estimation methods to learn the range of appearances that the target exhibits in the new, lowdimensional coordinate system. Because of the lower dimensionality, obtaining a useful estimate of either the PDF or the interclass discriminant function requires relatively few examples.

An important variation on this methodology is discriminativemodels, which attempt to model the differences betw een classes rather than the classes themselves. Such models can often be learned more efficiently and accurately than by directly modeling the PDF. A simple linear example of such a difference feature is the Fisher discriminant. Systems can also employ discriminant classifiers, such as support vector machines, which attempt to maximize the margin between classes.

\section{FACE RECOGNITION EFFORTS}

The subject of face recognition is as old as computer vision because of the topic's practical importance and theoretical interest from cognitive scientists. Despite the fact that other identification methods (such as fingerprints or iris scans) can be more accurate, face recognition has always been a major research focus because it is noninvasive and seems natural and intuitive to users.

Perhaps the most famous early example of a face recognition system is that of Teuvo Kohonen of the Helsinki University of Technology, ${ }^{4}$ who demonstrated that a simple neural net could perform face
Face recognition

capitalizes on

regularities that are

peculiar to humans. 


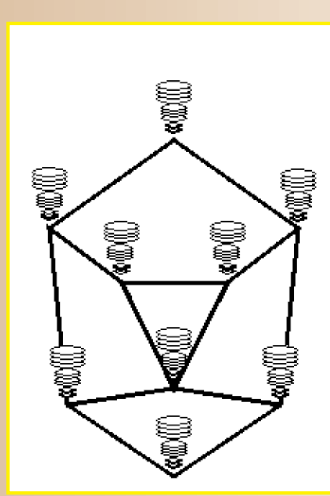

(a)

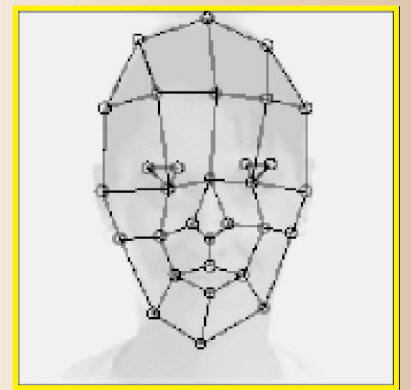

(b)
Figure 1. USC's system uses elastic graph matching for face recognition. It creates a face bunch graph from 70 face models to obtain a general representation called (a) an object-adapted grid. The system then (b) matches a given image to the face bunch graph to find the fiducial points. It creates an image graph using elastic graph matching and then compares that image to a database of faces for recognition.

recognition for aligned and normalized images of faces. The network he employed computed a face description by approximating the eigenvectors of the image's autocorrelation matrix. These eigenvectors are now known as eigenfaces.

Kohonen's system was not a practical success, however, because it relied on precise alignment and normalization. In the following years, many researchers tried face recognition schemes based on edges, interfeature distances, and other neural-net approaches. W hile several were successful with small databases of aligned images, none successfully addressed the more realistic problem of large databases where the face's location and scale are unknown.

M ichael Kirby and Lawrence Sirovich of Brown University 5 later introduced an algebraic manipulation that made it easy to directly calculate the eigenfaces. They also showed that it required fewer than 100 eigenfaces to accurately code carefully aligned and normalized faceimages. $M$ atthew Turk and Alex Pentland of $\mathrm{M} \mathrm{IT}^{6}$ then demonstrated that the residual error when coding with the eigenfaces could be used to detect faces in cluttered natural imagery and to determine the precise location and scale of faces in an image. They then demonstrated that coupling this method for detecting and localizing faces with the eigenface recognition method could achieve reliable, real-time recognition of faces in a minimally constrained environment. This demonstration that a combination of simple, real-time pattern recognition techniques could create a useful system sparked an explosion of interest in face recognition.

Cur rent work

By 1993, researchers claimed that several al gorithms provided accurate performance in minimally constrained environments. To better understand the potential of these algorithms, the US D efense Ad-vanced
Research Projects Agency and the US Army Research Laboratory established the Feret (face recognition technology) program with the goals of evaluating their performance and encouraging advances in the technology. ${ }^{7}$

Feret identified three algorithms that demonstrated the highest level of recognition accuracy with large databases (1,196 people or more) under double-blind testing conditions: those of the University of Southern California (USC), illustrated in Figure 1; 8 the University of $M$ aryland (UM D); ${ }^{9}$ and the $M$ assachusetts Institute of Technology (M IT) M edia Laboratory, illustrated in Figure 2. ${ }^{10} \mathrm{O}$ nly two algorithms, those from USC and M IT, are capable of both minimally constrained detection and recognition; the UMD system requires approximate eye locations to operate. Rockefeller University developed a fourth al gorithm, illustrated in Figure 3, that was an early contender, but it was withdrawn from testing to form a commercial enterprise. ${ }^{11}$ The M IT and USC algorithms have also become the basis for commercial systems.

The M IT, Rockefeller, and UM D algorithms all use versions of the eigenface transform followed by discriminative modeling. The UM D algorithm uses a linear discriminant, while the M IT system employs a quadratic discriminant. The R ockefeller system uses a sparse version of the eigenface transform follow ed by a discriminative neural network. The USC system, in contrast, takes a very different approach. It begins by computing Gabor jets from the image and then does a flexible template comparison of image descriptions using a graph-matching algorithm.

The Feret database testing employs faces with variable positions, scales, and lighting in a manner consistent with mug shot or driver's license photography. W ith databases of fewer than 200 people and images taken under similar conditions, all four algorithms perform nearly perfectly. Interestingly, even simple correlation matching can sometimes achievesimilar accuracy for databases of only 200 people.7 This is strong evidence that any new algorithm should be tested with databases of at least 200 individuals and should achieve performance over 95 percent on mug-shot-likeimages to beconsidered potentially competitive.

In the larger Feret testing (with 1,196 or more images), the performance of the four algorithms are similar enough that it is difficult or impossible to make meaningful distinctions among them (especially if adjustments for date of testing are made). W ith frontal images taken on the same day, the typical first-choice recognition performance is 95 percent accuracy. For images taken with different cameras and lighting, typical performance drops to 80 percent accuracy. For images taken one year later, the typical accuracy is approximately 50 percent. Still, it should benoted that even 50 percent accuracy is hundreds of times chance performance. 
1. The system collects a database of face images.

2. It generates a set of eigenfaces by performing principal component analysis (PCA) on the face images. Approximately 100 eigenvectors are enough to code a large database of faces.

3. The system then represents each face image as a linear combination of the eigenfaces.

4. Given a test image, the system approximates it as a combination of eigenfaces. A distance measure indicates the similarity between two images.

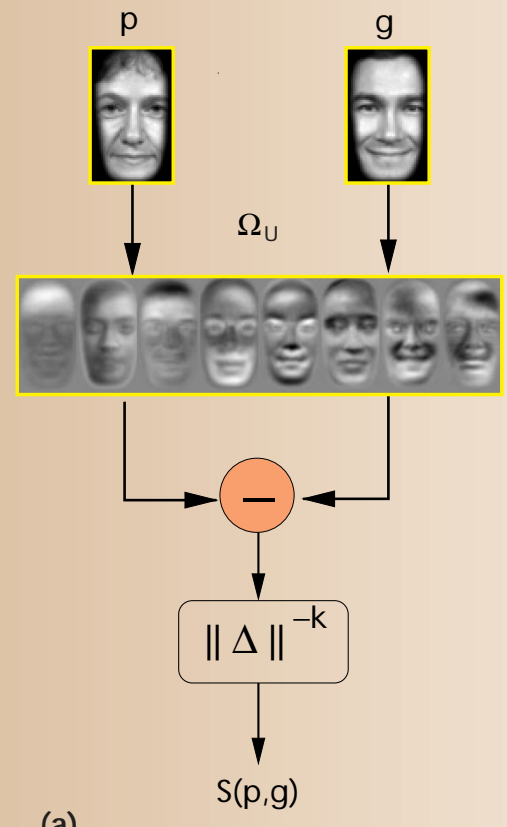

(a)

Figure 2. MIT's system of using eigenfaces for face recognition relies on (a) appearance and (b) discriminative models.

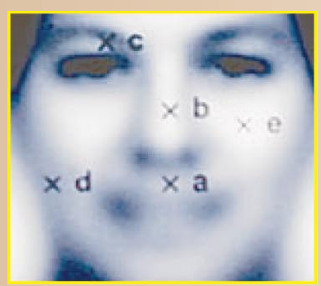

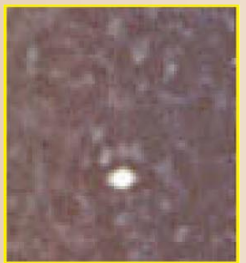

(a)

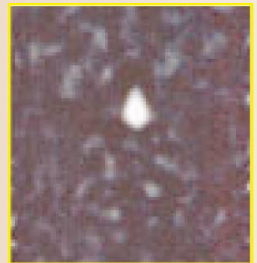

(b)

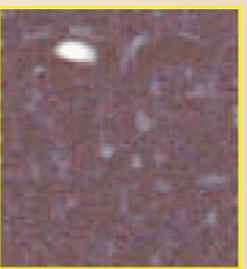

(c)

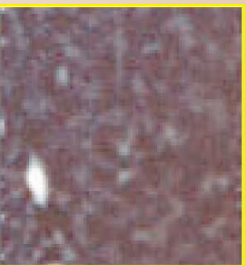

(d)

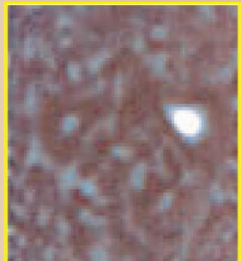

(e)

Figure 3. Rockefeller University's system of using local feature analysis for face recognition. The parts marked on the image to the left correspond to receptive fields for the (a) mouth, (b) nose, (c) eyebrow, (d) jawline, and (e) cheekbone. (Reprinted with permission of NYT Pictures) 


\section{Growing numbers of applications are using face recognition as the initial step toward interpreting human actions.}

\section{Commer cial systems and applications}

Several face recognition products are commercially available. Algorithms developed by the top contenders in the Feret competition are the bases for some available systems; others were developed outside of the Feret testing framework. While it is extremely difficult to judge, three systems-from Visionics, Viisage, and $M$ iros- seem to be the current market leaders.

- Visionics' Facelt software is based on the local featureanalysis al gorithm developed at Rockefeller University. A commercial company in the UK is incorporating Facelt into a closedcircuit television anticrime system called $M$ andrake. This system searches for known criminals in video data acquired from 144 closed-circuit camera locations. When a match occurs, the system notifies a security officer in the control room.

- Viisage, another leading face recognition company, uses the eigenface-based recognition algorithm developed at the M IT M edia Laboratory. Companies and government agencies in many US states and several developing nations use Viisage's system in conjunction with identification cardsfor example, driver's licenses and similar government ID cards.

- M iros uses neural network technology for its TrueFace face recognition software. TrueFace is used by the M r. Payroll Corp. system in its check cashing system and has been deployed at casinos and similar sites in many US states.

\section{NOVEL APPLICATIONS}

Face recognition systems are no longer limited to identity verification and surveillance tasks. Growing numbers of applications are using face recognition as the initial step toward interpreting human actions, intentions, and behavior as a central part of next-generation smart environments. $M$ any actions and behaviors humans display can only be interpreted if you also know the identities of the individuals and the people around them. Examples are a valued repeat customer entering a store, behavior monitoring in an elder care or child care facility, and command-and-control interfaces in a military or an industrial setting. In each of these applications, identity information is crucial to providing machines with the background knowledge needed to interpret measurements and observations of human actions.

\section{Face recognition for smart envir onments}

Researchers today are actively building smart environments-visual, audio, and haptic interfaces with environments such as rooms, cars, and office desks. ${ }^{1,2}$
In these applications, a key goal is to give machines perceptual abilities that allow them to function naturally with people- to recognize people and remember their preferences and peculiarities, to know what they are looking at, and to interpret their words, gestures, and unconscious cues, such as vocal prosody and body language. Researchers are exploring applications for these perceptually aw are devices in health care, entertainment, and collaborative work.

Facial-expression recognition interacts with other smart-environment capabilities. For example, a smart system should know whether the user looks impatient-because information is being presented too slowly-or confused because it is coming too fast. Facial expressions provide cues for identifying and distinguishing between these states. Recently, much effort has gone into creating a person-independent expression recognition capability. While thereare similarities in expressions across cultures and between people, for anything but the most gross facial expressions, the analysis must account for the person's normal resting facial state- something that definitely isn't the same between people. Consequently, facial-expression research has so far been limited to recognition of a few discrete expressions rather than addressing the entire spectrum of expressions along with their subtle variations. Before a system can achieve a really useful expression analysis capability, it must first be able to recognize and tune its parameters to a specific person.

\section{Wear able recognition systems}

When we build computers, cameras, microphones, and other sensors into a person's clothes, the computer's view moves from a passive third-person to an active first-person vantage point. ${ }^{3}$ These wearable devices can adapt to a specific user and be more intimately and actively involved in the user's activities. The wearable-computing field is rapidly expanding and just recently became a full-fledged technical committee within the IEEE Computer Society. Consequently, we can expect to seerapidly growing interest in the largely unexplored area of first-person image interpretation.

Face recognition is an integral part of wearable systems like memory aids and context-aw are systems. Thus, developers will integrate many future recognition systems with clothing and accessories. For instance, if you build a camera into your eyeglasses, then face recognition software can help you remember the name of the person you are looking at by whispering it in your ear. The US Army has started testing such devices for use by border guards in Bosnia, and researchers at the University of R ochester's Center for Future $\mathrm{H}$ ealth are looking at them for patients with A Izheimer's disease (see http://wearables.www. media.mit.edu/projects/wearables and http://www. futurehealth.rochester.edu). 


\section{FUTURE WORK}

Today's face recognition systems work very well under constrained conditions, such as frontal mug shot images and consistent lighting. All current face recognition algorithms fail under the vastly varying conditions in which humans can and must identify other people. $\mathrm{N}$ ext-generation recognition systems will need to recognize people in real time and in much less constrained situations.

We believe that identification systems that are robust in natural environments-in the presence of noise and illumination changes- cannot rely on a single modality; thus, fusion with other modalities is essential. Technology used in smart environments has to be unobtrusive and allow users to act freely. Wearable systems in particular require the sensing technology to be small, low power, and easily integrable with clothing. Considering all the requirements, systems that use face and voice identification seem to have the most potential for widespread application.

Cameras and microphones today are very small and lightweight, and have been successfully integrated with wearable systems. Audio- and video-based recognition systems have the critical advantage of using the modalities humans use for recognition. Finally, researchers are beginning to demonstrate that unobtrusive audioand video-based personal identification systems can achieve high recognition rates without requiring the user to be in a highly controlled environment. ${ }^{12}$

$\mathrm{T}$ he goal of smart environments is to create a space where computers and machines are more like helpful assistants, rather than inanimate objects. Face recognition technology could play a major part in achieving this goal, and it has come a long way in the past 20 years. But to achieve the goal of widespread application in smart environments, next-generation face recognition systems will have to fit naturally within the pattern of normal human interactions and conform to human intuitions about when recognition is likely. This implies that future smart environments should use the same modalities as humans and have approximately the same limitations. Although substantial research remains to be done, these goals now appear to be within reach.

\section{References}

1. M. Weiser, "The Computer for the 21st Century," Scientific A merican, M ar. 1991, pp. 66-76.

2. A. Pentland, "Smart Rooms, Smart Clothes," Scientific A merican, A pr. 1996, pp. 68-76.

3. A. Pentland, "Wearable Intelligence," Scientific A merican Presents, A pr. 1998, pp. 90-95.

4. T. Kohonen, Self-O rganization and Associative M emory, Springer-Verlag, Berlin, 1989.
5. M. Kirby and L. Sirovich, "A pplication of the KarhunenLoeve Procedure for the Characterization of $\mathrm{H}$ uman Faces," Trans. IEEE Pattern Analysis and Machine Intelligence, Jan. 1990, pp. 103-108.

6. M. Turk and A. Pentland, "Eigenfaces for Recognition," J. Cog. N euroscience, Jan. 1991, pp. 71-86.

7. P. Phillips et al., "The Feret Database and Evaluation Procedure for Face Recognition A Igorithms," I mage and Vision Computing, M ay 1998, pp. 295-306.

8. L. Wiskott et al., "Face Recognition by Elastic Bunch Graph M atching," Trans. IEEE Pattern Analysis and M achine Intelligence, July 1997, pp. 775-779.

9. K. Etemad and R. Chellappa, "Discriminant Analysis for Recognition of H uman Face Images," J. O ptical Soc. of America, pp. 1724-1733.

10. B. M oghaddam and A. Pentland, "Probabilistic Visual Recognition for O bject Recognition," Trans. IEEE Pattern Analysis and M achine Intelligence, July 1997, pp. 696-710.

11. P. Penev and J. A tick, "Local Feature A nalysis: A General Statistical Theory for O bject R epresentation," N etwork: Computation in N eural Systems, M ar. 1996, pp. 477-500.

12. T. Choudhury et al., "M ultimodal Person Recognition Using Unconstrained Audio and Video," Proc. 2nd Conf. Audio- and Video-Based Biometric Person Authentication, Univ. of M aryland, College Park, M d., 1999, pp. 176-181.

Alex (Sandy) Pentland is academic head of the M IT M edia L aboratory, Toshiba professor, and codirector of the $\mathrm{C}$ enter for Future $\mathrm{H}$ ealth. $\mathrm{He}$ is interested in the development of new technologies that empower people, including thosefrom developing nations. Pentland has a PhD from MIT. He is a cofounder of the IEEE Face and G esture R ecognition Conference and the IEE E Computer Society Technical Committee on Wearable Information Devices. Contact him at sandy@media.mit.edu.

Tanzeem Choudhury is a graduate student at the M IT $M$ edia $L$ aboratory. $H$ er research inter ests include face recognition, real-time multimodal person identification, and facial expression analysis. Choudhury has an SM from MIT and a BS in electrical engineering from the U niversity of Rochester. Contact her at tanzeem@media.mit.edu. 